\title{
Propagation Properties of the Precipitation Band in an $\mathrm{AlCl}_{3} / \mathrm{NaOH}$ System
}

Takahiko Ban $^{\text {a,*, Yuichiro Nagatsu }}{ }^{\mathrm{b}}$ and Hideaki Tokuyama ${ }^{\mathrm{b}}$

${ }^{a}$ Division of Chemical Engineering, Department of Materials Engineering Science,

Graduate School of Engineering Science, Osaka University, Machikaneyamacho 1-3,

Toyonaka, Osaka 560-8531, Japan

b Department of Chemical Engineering, Tokyo University of Agriculture and Technology, 2-24-16 Naka-cho, Koganei, Tokyo 184-8588, Japan

*Corresponding author

Takahiko Ban

Tel: +81-6-6850-6625, Fax: +81-6-6850-6625

E-mail: $\underline{\text { ban@cheng.es.osaka-u.ac.jp }}$ 


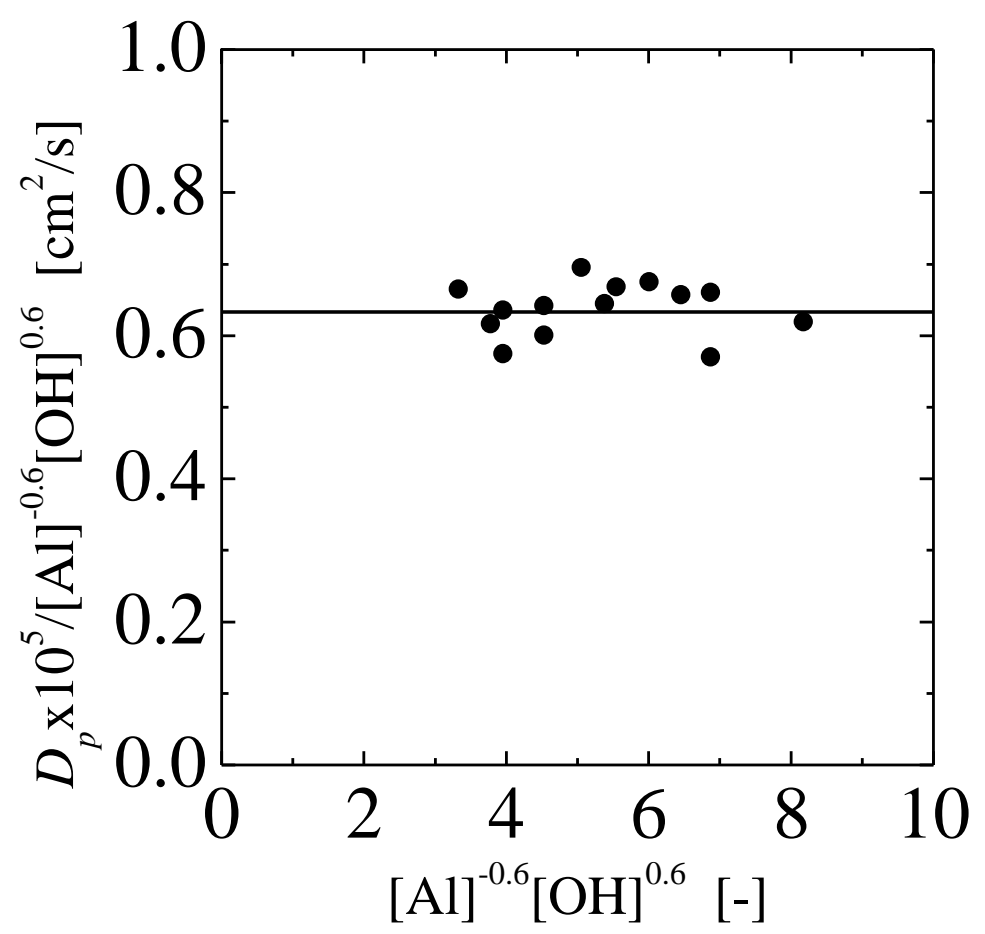

Figure S1. Relation between $D_{p} /\left[\mathrm{Al}^{3+}\right]^{-0.6}\left[\mathrm{OH}^{-}\right]^{0.6}$ and $\left[\mathrm{Al}^{3+}\right]^{-0.6}\left[\mathrm{OH}^{-}\right]^{0.6}$. 


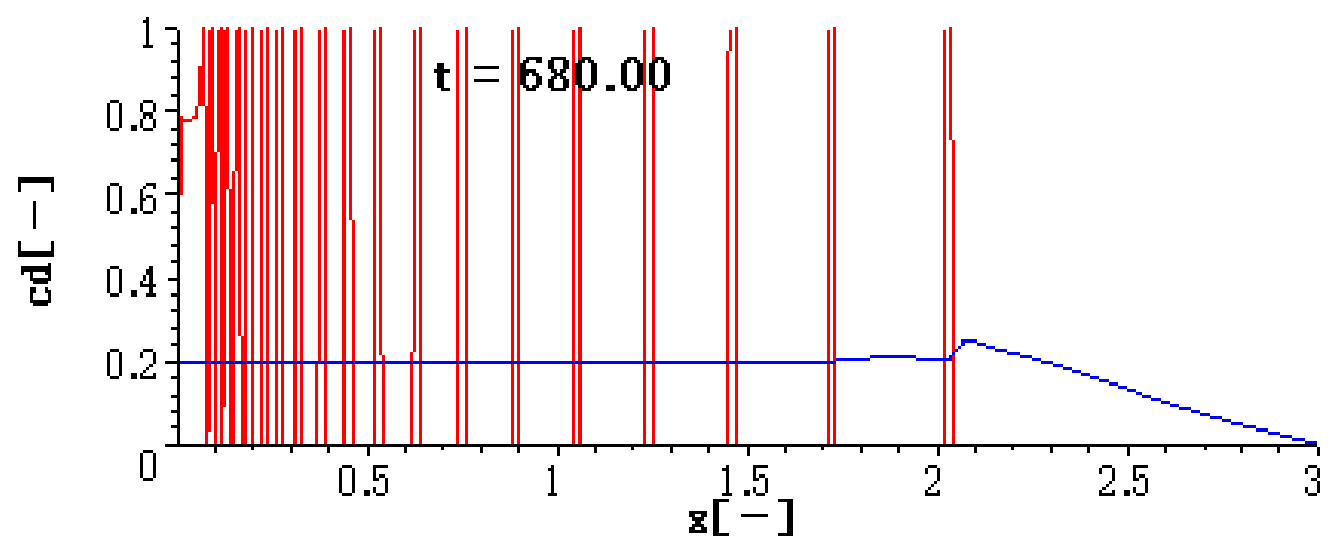

Figure S2. Model simulation of the Liesegang bands: $D_{a}=0.001, D_{b}=0, D_{c}=0.001, C_{s}$ $=0.2, c_{s s}=0.4, k_{1}=50, k_{2}=0, q=50, a_{0}=10$, and $b_{0}=1$. The blue and red curves represent the concentration distributions of the colloid and the precipitate, respectively. 
(a)

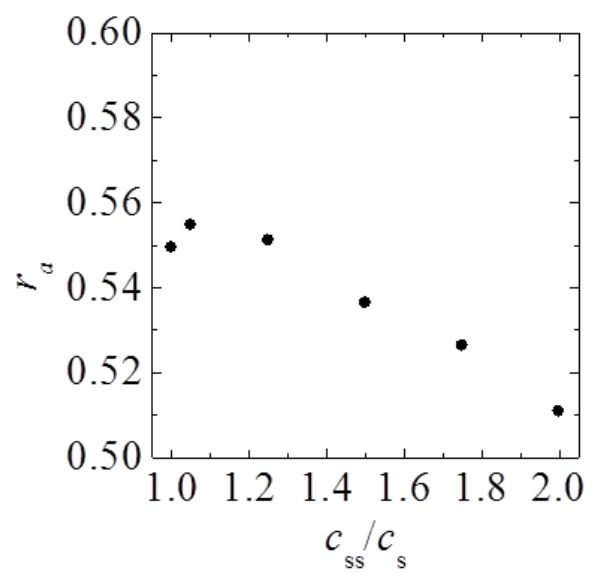

(b)

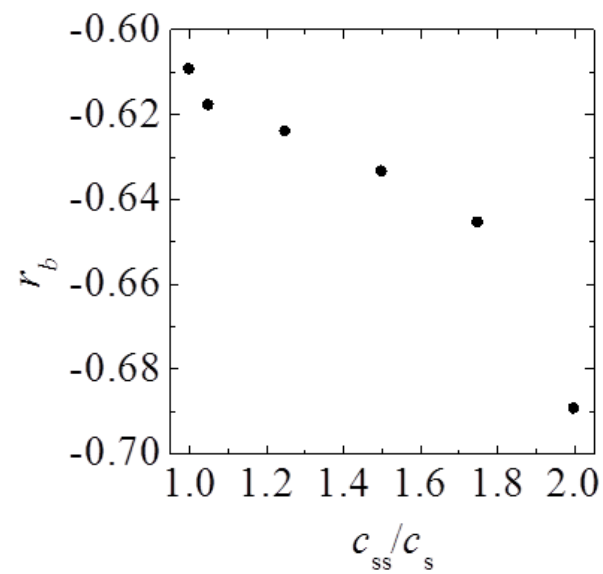

Figure S3. Relationship between the exponents $r_{a}$ and $r_{b}$ of eq. 5 and the ratio of supersaturation concentration to saturation concentration. The values of the parameters are: $D_{a}=0.001, D_{b}=0, D_{c}=0.001, C_{s}=0.2, k_{1}=50, k_{2}=0.5, q=50$. 
(a)

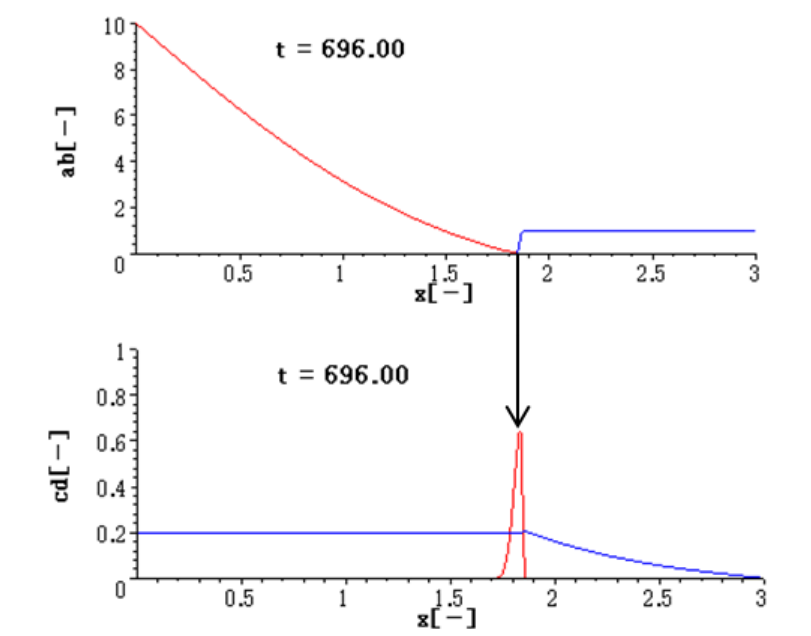

(c)

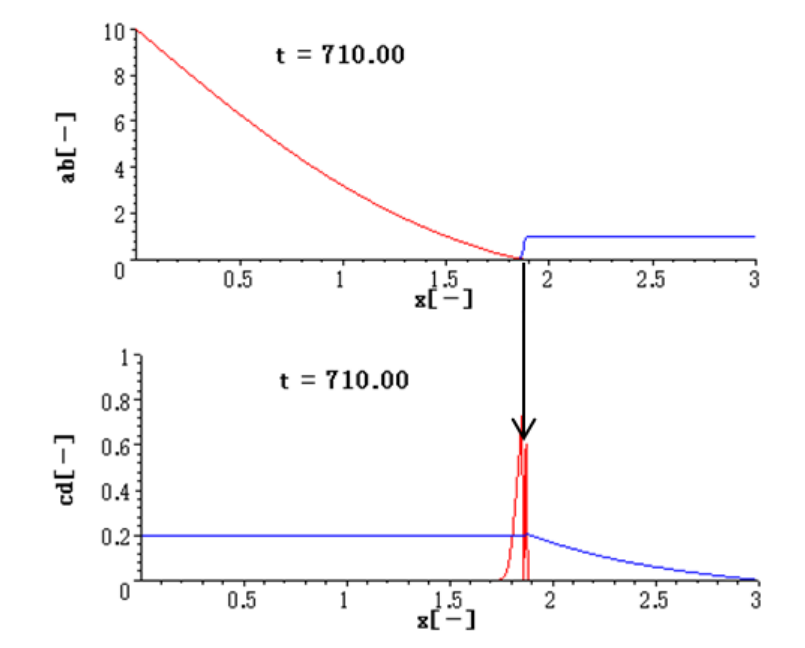

(b)
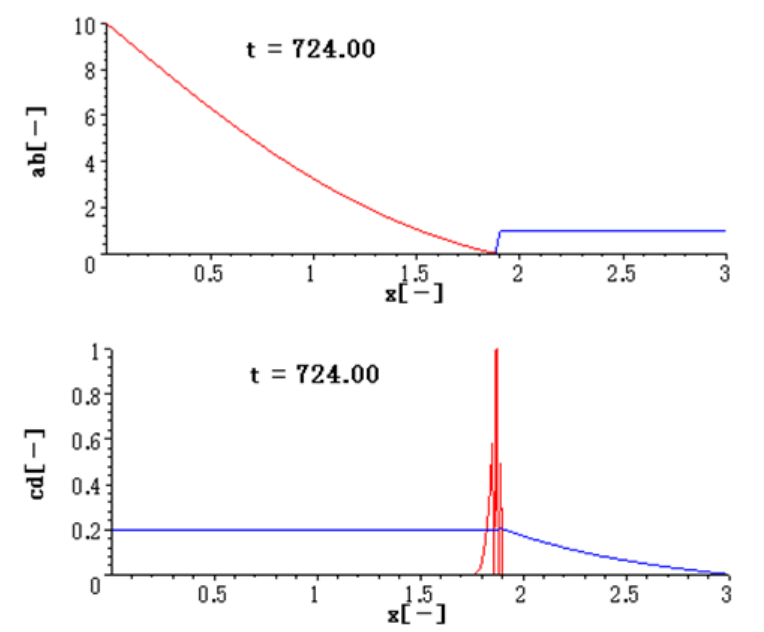

(d)
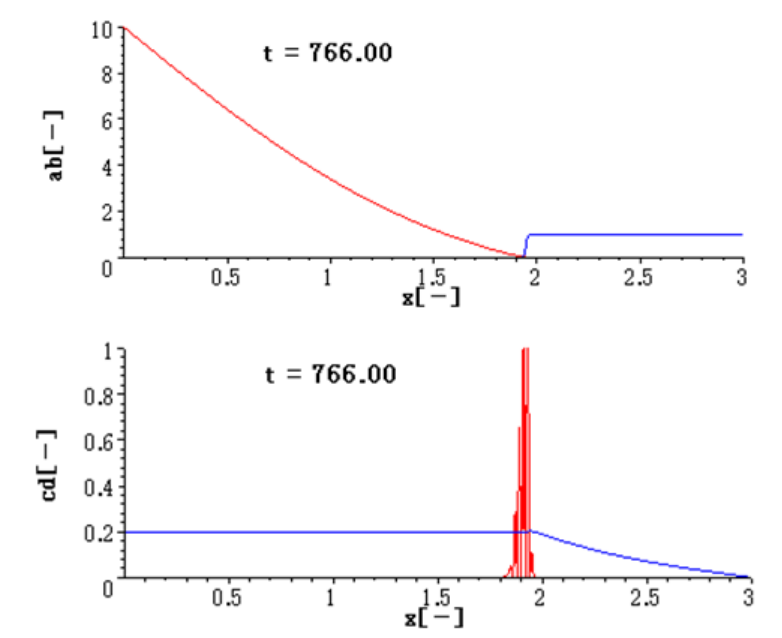

Figure S4. Simulated dynamics of multiple discrete bands. The blue and red curves represent the concentration distributions of the outer (colloid) and the inner electrolytes (precipitate) in the upper panels (in the lower panels), respectively. (a) $t=696$ (b) $t=$ 710 , (c) $t=724$, and (d) $t=766$. The supersaturation concentration is set at $c_{s s}=0.21$, and the values of the other parameters are the same as those in Figure 4. 
(a)

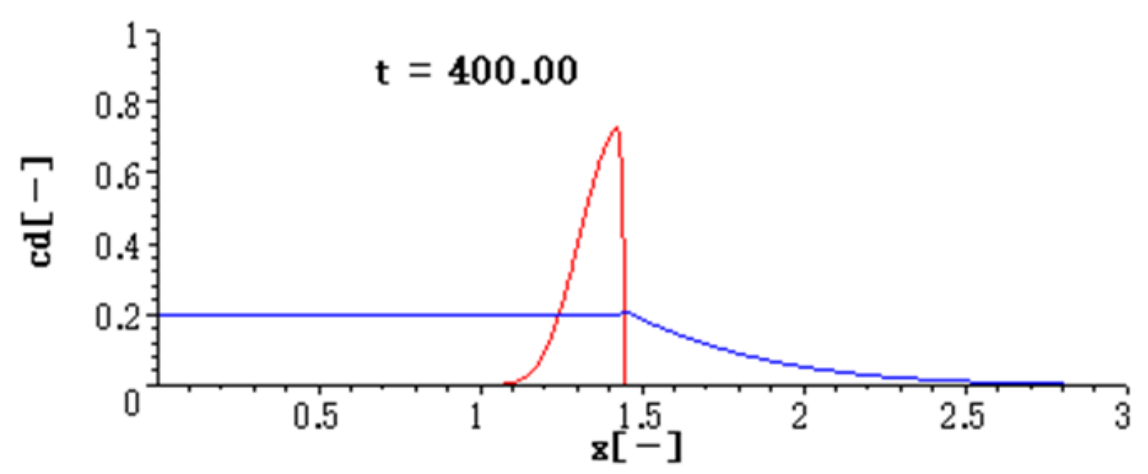

(b)

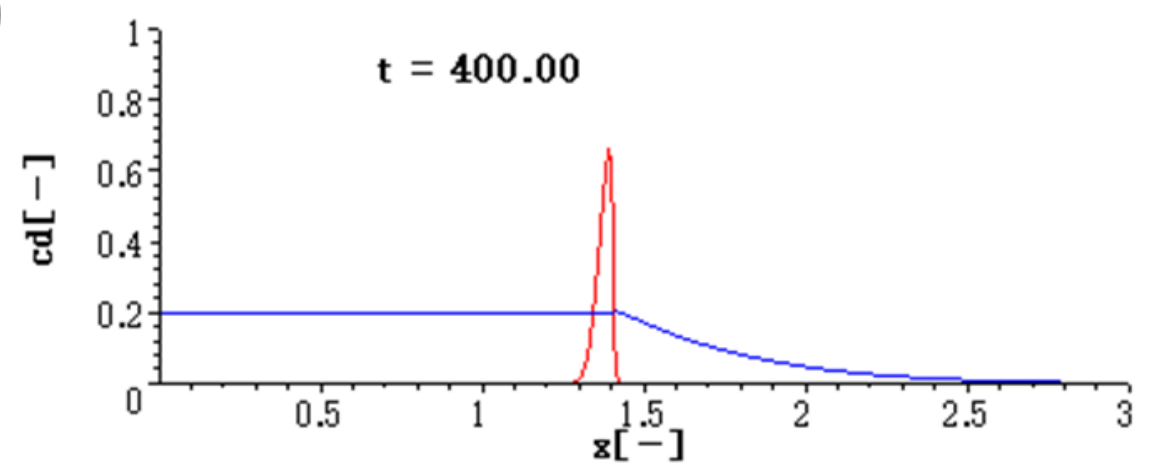

(c)

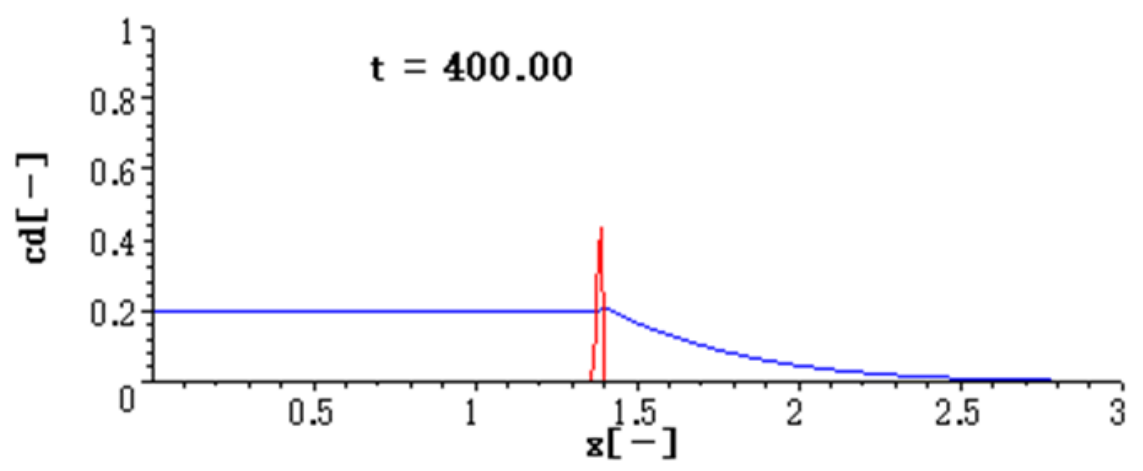

Figure S5. Effect of the rate constant of precipitate formation $q$ on the dynamics of propagating precipitation band. (a) $q=5$, (b) $q=10$, and (c) $q=100$. The blue and red curves represent the concentration distributions of colloid and inner precipitate, respectively. The values of the parameters are: $D_{a}=0.001, D_{b}=0, D_{c}=0.001, c_{s}=0.2$, $c_{s s}=0.21, k_{1}=50, k_{2}=0.5$. 
(a)

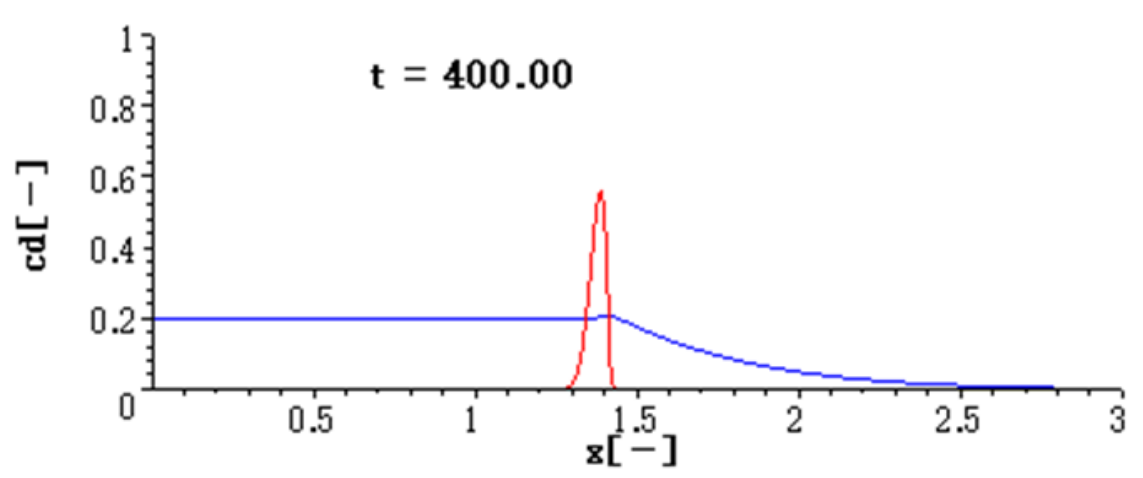

(b)

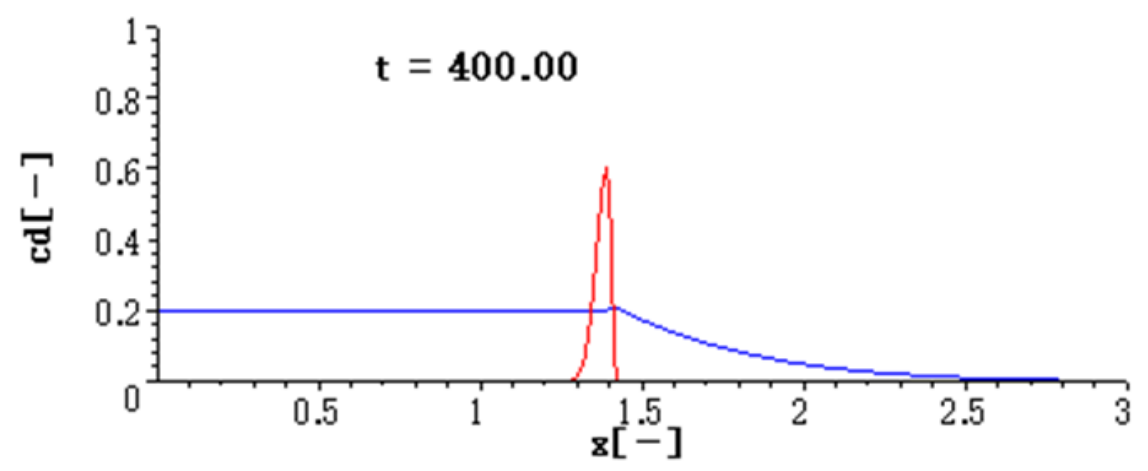

(c)

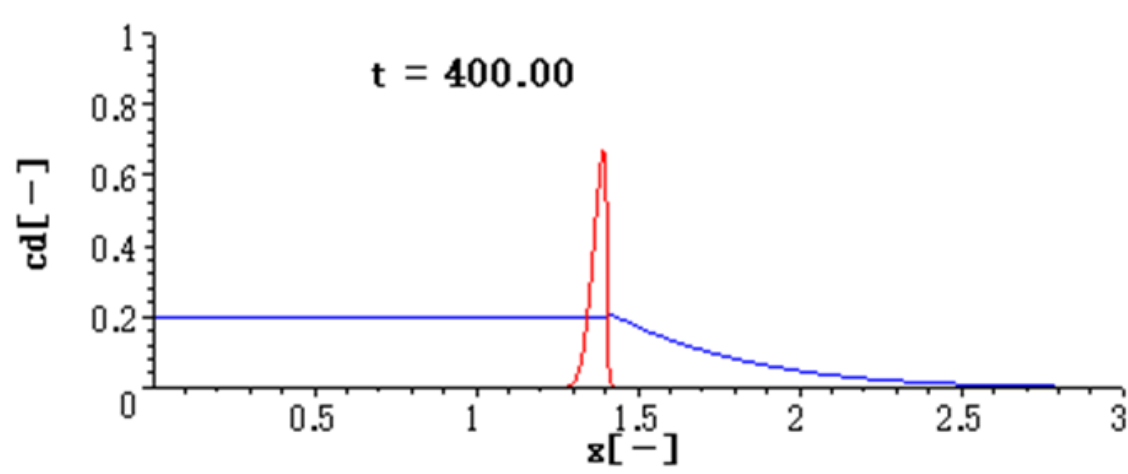

Figure S6. Effect of the rate constant of redissolution $k_{2}$ on the dynamics of the propagating precipitation band. (a) $k_{2}=0.05$, (b) $k_{2}=0.5$, and (c) $k_{2}=5$. The blue and red curves represent the concentration distributions of the colloid and the precipitate, respectively. The values of the parameters are: $D_{a}=0.001, D_{b}=0, D_{c}=0.001, c_{s}=0.2$, $c_{s s}=0.21, k_{1}=50, q=50$. 